\title{
Acción colectiva y educación popular: contribuciones para un conocimiento emancipatorio
}

\section{Collective action and popular education: contributions for emancipatory knowledge}

\section{Resumen}

Ricardo Delgado S. ${ }^{1}$

El texto recoge los planteamientos expuestos en el Congreso Internacional Vecindades y Fronteras en abril de 2010, en Bogotá. Acogiendo los referentes centrales expuestos por B. De Sousa en su empeño por renovar la teoría crítica y reinventar la emancipación social, el artículo presenta una reflexión acerca de la invención y actualización de un conocimiento emancipatorio desde la acción colectiva que emprenden los movimientos sociales en el contexto de América Latina. De igual manera, es de su interés destacar algunas relaciones y retos que se establecen entre los movimientos sociales y los procesos de educación popular, respecto a la necesidad de potenciar una pedagogía crítica de la traducción cultural que propicie el diálogo y el intercambio de una ecología de los saberes entre los movimientos sociales, las ONGs y los científicos sociales, generando espacios de aprendizaje colectivo que revitalicen las acciones sociales y las redes locales y globales de conocimiento orientado hacia la trasformación social.

\section{Palabras clave:}

Acción colectiva, movimientos sociales, educación popular, conocimiento emancipatorio.

\section{Abstract}

The text includes the issues outlined in the International Congress of Neighborhoods and Borders in April 2010 in Bogotá. Welcoming the central referents presented by B. De Sousa in their efforts to renew critical theory and reinvent social emancipation, the article presents a reflection about the invention and gives an update of an emancipatory knowledge from the collective action undertaken by social movements in the Latin American context. Similarly, it is of interest to highlight some relationships and challenges that arise between social movements and processes of popular education on the need to promote critical pedagogy of cultural translation that promotes dialogue and exchange of an ecology of knowledge between social movements, NGOs and social scientists, generating collective learning spaces to revitalize the social actions and the local and global networks of knowledge-oriented social transformation.

\section{Keywords:}

Collective action, social movements, popular education, emancipatory knowledge.

Artículo recibido el 29 de julio de 2010 y aprobado el 28 de marzo de 2011

1 Doctor en Ciencias Sociales, Niñez y Juventud, Universidad de Manizales-CINDE, docente-investigador de la Facultad de Educación de la Pontificia Universidad Javeriana, Bogotá. rdelgado@javeriana.edu.co 


\section{Introducción}

El propósito del presente artículo se enmarca en la reflexión y problematización acerca de la invención social de un conocimiento emancipatorio, teniendo como referentes centrales algunos de los planteamientos de Boaventura De Sousa Santos presentados en sus libros Crítica de la razón indolente: contra el desperdicio de la experiencia y Renovar la teoría crítica y reinventar la emancipación social. En esta oportunidad la pregunta por la reinvención de la emancipación social se abordara desde la acción colectiva, si bien reconocemos la diversidad de expresiones que esta conlleva, nos referiremos a los movimientos sociales ${ }^{2}$ en el contexto de América Latina. Ligado a lo anterior, es de nuestro interés destacar algunas relaciones y retos que los movimientos sociales, como fuentes generadoras de conocimiento emancipatorio, le plantean a la educación popular como práctica educativa respecto a su horizonte de acción política. De igual manera se hará referencia a la contribución de la educación popular en la construcción y renovación de un pensamiento crítico emancipador para el momento actual del continente latinoamericano.

Para tales propósitos el texto se organiza en dos apartados. El primero busca destacar el protagonismo social y político que los movimientos y las organizaciones sociales han adquirido en el continente latinoamericano, destacando su capacidad de reflexividad, solidaridad y cambio como expresiones que sustentan un conocimiento alternativo y emancipatorio. En un segundo momento resaltaremos cómo estas experiencias de acción colectiva que pretenden constituir subjetividades rebeldes, como diría B. De Sousa, le plantean desafíos a la

2 Para efectos de la presente disertación acojo el planteamiento que nos propone M. R. Goldar (2008, p. 6) quien concibe los movimientos sociales "como aquellas conformaciones que, con distintos grados de consolidación y con alguna permanencia en el tiempo, se estructuran en torno a intereses comunes y a un fuerte componente identitario; que emergen en la sociedad con alguna capacidad de colocar temas, demandas, propuestas, etc. que no son tenidas en cuenta por el orden social vigente. En ese sentido son fuertemente disruptivos y encarnan la posibilidad de desarrollar procesos de transformación social". educación popular, y cómo ésta, desde su tradición teórico práctica, contribuye a la consolidación de un pensamiento crítico sobre el cual se sustente la emergencia y consolidación de una pedagogía de la traducción cultural, que desde una perspectiva intercultural y poscolonial de nuestras acciones colectivas, conlleve paulatinamente a establecer agendas comunes que propicien la articulación e integración de los sujetos sociales.

\section{Acción colectiva y subjetividad: un abordaje desde el conocimiento como emancipación}

En su propósito por renovar la teoría crítica y reinventar la emancipación social, B. De Sousa parte por adelantar una crítica a la ciencias sociales, destacando como uno de los problemas relevantes la discrepancia profunda entre teoría y práctica social, situación que ha conducido a que se consolide una teoría ciega que invisibiliza, desprecia, desacredita experiencias sociales debido a que muchas de ellas se llevan a cabo en contextos remotos y distintos de las instancias hegemónicas e institucionalizadas de la ciencia moderna, que ha privilegiado un conocimiento como regulación, el cual ha pervertido y limitado las posibilidades emancipatorias que pueden estar presentes en otros tipos de conocimientos y saberes ligados con las prácticas sociales.

Es así como expresiones sociales no conocidas ni legitimadas por las ciencias sociales, que se desenvuelven en contextos locales, son hostilizadas, ignoradas, generándose un desperdicio por la riqueza inagotable de experiencias sociales que tienen lugar en contextos periféricos, como es el caso de Latinoamérica, donde se destacan acciones colectivas orientadas a crear innovaciones democráticas, formas de economía solidaria y social, procesos de constitución de ciudadanía en el contexto de demandas de derechos culturales, ambientales, de territorio, entre otros; donde los movimientos y las organizaciones sociales -indígenas, campesinos, feministas, ambientalistas, juveniles, obrerosjuegan un vital protagonismo para promover un 
conocimiento social emancipador desde el sur, que haga posible un marco de articulación entre los sujetos sociales.

Enfrentar esta situación y confrontar esta razón indolente, que se considera única, exclusiva y que no se ejercita lo suficiente para poder mirar la riqueza de otras epistemologías, requiere con urgencia reinventar las ciencias sociales, para hacer que estas sean parte de la solución y no del problema, y de esta manera resistir la contracción del presente, la reducción de la diversidad cultural y poder así demostrar la presencia de otros saberes, conocimientos y realidades, lo que hace necesario postular, en términos de B. De Sousa (2006, p. 23), un conocimiento, una sociología de las ausencias que se plantea "como un procedimiento transgresivo, una sociología insurgente para intentar mostrar que lo que no existe es producido activamente como no existente, como una alternativa no creíble, como una alternativa descartable, invisible a la realidad hegemónica del mundo".

Por lo anterior, una sociología de las ausencias, de los conocimientos ausentes, parte de la premisa de que las prácticas sociales son prácticas de conocimientos, como las que promueven los movimientos sociales en nuestros contextos urbanos y rurales orientados a crear y afianzar solidaridad. La concreción de esta premisa para revitalizar el papel de los movimientos sociales como fuentes generadores de pensamiento crítico emancipatorio que propicie el diálogo y la articulación entre los sujetos sociales, requiere que se transite y se recurra a una forma de conocimiento que no reduzca la realidad, aquello que existe, es decir, es necesario un conocimiento que incorpore realidades suprimidas, silenciadas y marginadas.

Por otra parte, la invención de un renovado conocimiento emancipador que nutra el sentido común donde se forjan los vínculos de solidaridad ${ }^{3} \mathrm{y}$ reconocimiento entre los sujetos sociales como una

3 “La solidaridad es el conocimiento obtenido en el proceso, siempre inacabado, de volvernos más capaces de reciprocidad a través de la construcción y del reconocimiento de la intersubjetividad. El énfasis en la solidaridad convierte la comunidad en el campo privilegiado del conocimiento emancipador" (B. De Sousa S., 2003, p. 90). forma específica de saber, debe ser complementado por la invención de subjetividades individuales y colectivas desestabilizadoras, que tienen capacidad y deseos de revelarse contra prácticas sociales conformistas y rutinarias. De esta manera una sociología de los conocimientos y de los agentes ausentes, como lo formula B. De Sousa, tiene el propósito de hacer proliferar cualidades emergentes, provenientes de diferentes prácticas sociales, las cuales compiten y nutren el campo de disputa social y política entre acciones conformistas y acciones rebeldes.

Esta sociología de los conocimientos ausentes, como de los agentes ausentes, nos invita a revisar los límites de la representación en las ciencias sociales convencionales ${ }^{4}$ respecto al estudio de los movimientos sociales, con el propósito de revisar los criterios de relevancia, identificación, duración, interpretación y evaluación de sus trayectorias sociales y políticas.

La renovación de la representación de los límites en las ciencias sociales definidos por la primacía del conocimiento-regulación sobre el conocimientoemancipación, pasa por la sustitución y superación de los modos de producción de ausencias ${ }^{5}$ en la

4 B. de Sousa (2003) se refiere por los límites de la representación a aquellos procedimientos, criterios y parámetros que las ciencias establecen para definir la relevancia de un objeto de estudio, las escalas y perspectivas para su valoración, identificación, localización temporal, interpretación y evaluación.

5 B. De Sousa S. (2006) identifica cinco modos de producción de ausencias en la racionalidad occidental que han privilegiado un conocimiento-regulación. El primer modo es la monocultura del saber y del rigor, donde prima la idea de que el único saber riguroso es el saber científico y, por lo tanto, otros conocimientos no tienen la validez ni el rigor del conocimiento científico. Esta monocultura elimina muchas realidades y experiencias sociales que se basan en conocimientos populares, indígenas, campesinos, urbanos, que no son evaluados como importantes y por lo cual se invizivilizan y se desacreditan. La segunda monocultura es la del tiempo lineal, donde prevalece la idea de que la historia tiene un sentido, una dirección, y sobre el cual se asientan los conceptos de progreso, modernización, desarrollo y globalización, desconociendo otras nociones y percepciones de temporalidad y durabilidad. La tercera monocultura es la de la naturalización de las diferencias que ocultan jerarquías, de las cuales la clasificación racial, étnica, sexual, son en la actualidad las más persistentes. El cuarto modo de generación de ausencias es el establecimiento de la escala dominante, donde se privilegian como parámetros de identificación y valoración el universalismo y la globalización, dejando por fuera lo particular y lo local. El último modo se refiere a la monocultura del productivismo capitalista, donde la idea fuerza es que el crecimiento económico y la producción determinan la productividad del trabajo humano 0 de la naturaleza, desconociendo otros criterios y limites. 
racionalidad occidental, es decir, sustituir las monoculturas del saber y del rigor, del tiempo lineal, de la naturalización de las diferencias, del establecimiento de escalas dominantes y del productivismo capitalista, por las ecologías ${ }^{6}$ que B. De Sousa sugiere para superar y renovar los límites y criterios, estableciendo una perspectiva curiosa que haga posible un ángulo diferente que reconstituya los procesos creativos centrales de las ciencias sociales y permita un abordaje que enriquezca el presente, a partir de otros conocimientos, de otras percepciones del tiempo, de otras escalas de representación, de productividad y, por supuesto, de interpretación.

Ahora bien, ante la pregunta central que orienta la reflexión ¿qué aportes reconocemos en los movimientos sociales en la construcción y reinvención de un conocimiento como emancipación social?, nos vemos avocados a estar atentos a explorar las señales que nos anuncian la emergencia de un conocimiento que conlleva un potencial emancipatorio y que se expresa en el continente en formas de resistencia y de luchas sociales y políticas, que si bien por ahora se subscriben en el ámbito local y aun son embrionarias, contienen un marco de aspiración y de utopía de una nueva sociedad que está creando un campo de disputa, de confrontación tanto en el nivel epistemológico como político, que se manifiesta cuando el concomimiento como emancipación social reconoce como forma de ignorancia el colonialismo, y se plantea si es posible conocer creando solidari-

6 La sociología de las ausencias en su propósito de de sustituir las monoculturas, propone cinco ecologías (B. De Sousa 2006, pp. 26 29): 1) La ecología de los saberes, que abre la posibilidad de que la ciencia entre, no como monocultura, sino como parte de una ecología de conocimientos más amplia, donde el saber científico entre en diálogo con otros saberes que adquieren relevancia social y política, los cuales se producen y se movilizan por fuera del sistema escolar formal. 2) La ecología de las temporalidades, que permite que se reconozcan diversas percepciones y nociones de tiempo, espacio y territorialidad, que no se agotan en una concepción cronológica y lineal.3) La ecología del reconocimiento, que busca promover un proceso de descolonización cognitiva y afectiva para poder producir algo que distinga, en una diferencia, lo que es producto de las jerarquías y lo que no lo es. 4) La ecología de la trans-escala, que abre la posibilidad de valorar los contextos locales, regionales, nacionales y globales en los que se insertan las acciones colectivas emprendidas por los agentes sociales. 5) La ecología de las productividades, que pretende recuperar y valorar los procesos alternativos de producción de las organizaciones económicas populares. dad, y desde ahí asumir el reto de democratizar la democracia.

Asumir una sociología de las emergencias como perspectiva de aproximación a los movimientos sociales en América Latina, como lo sugiere B. De Sousa, quizás implique comprender e interpretar sus acciones colectivas considerando varios planos de análisis donde podamos identificar las resistencias que hoy se dan en el continente:

\section{La acción colectiva como fuente generadora de diferentes lenguajes, nuevas narrativas, renovados imaginarios de problemas y de soluciones}

Este plano nos llevaría a reconocer en los movimientos sociales su capacidad de reflexividad, la cual radica en producir una controversia respecto de un estado de cosas cuya legitimidad y sentido normativo se dan por hechos. Este atributo hace que los movimientos sociales sean concebidos como agencias de significación colectiva que difunden nuevos significados en la sociedad.

De lo anterior se desprende su capacidad para crear marcos de acción colectiva, es decir nuevas narrativas, relatos de injusticia que agravian su dignidad, relatos de inclusión y de reconocimiento donde los valores de equidad y solidaridad actúan como cimientos desde los cuales justifican y legitiman ética y políticamente sus reivindicaciones, sus expresiones de resistencia orientadas a demandar una sociedad más justa e incluyente y la profundización de una democracia de alta intensidad, poniendo de presente la exigibilidad de nuevos derechos fundamentales: el derecho al agua, a la tierra, a la energía, el derecho a la biodiversidad y a los recursos naturales.

Sobre este aspecto coincido con M. R. Goldar (2009, pp. 71-72), quien afirma que más que la eficacia del movimiento, lo que amerita análisis,

es su capacidad y potencialidad por colocar temas y reivindicaciones en las agendas políticas de nuestros países, pero también por la novedad en sus repertorios, luchas que no sólo puedan ser eficaces como acción de protesta, sino fundamentalmente por lo que estas luchas colocan simbóli- 
camente en la escena pública, posesionando con fuerza nuevas formas de acción, que constituyen nuevos actores sociales, que entrañan renovados imaginarios sociales.

El reconocimiento de esta dimensión de análisis nos permite resaltar el postulado central de la ecología de los saberes, el cual radica en reconocer que las acciones colectivas que animan distintos movimientos sociales, son prácticas de conocimiento que por supuesto dialogan y se nutren del saber popular, del saber indígena, del saber de las poblaciones urbanas marginales y del saber científico.

\section{La acción colectiva como nueva forma de sociabilidad y de solidaridad}

Lo que diferencia a un movimiento social de otras expresiones de acción colectiva que podemos caracterizar como de simple agregación, es que los MS conllevan procesos de solidaridad, esto es, la capacidad que tienen sus integrantes de reconocerse a sí mismos y de ser reconocidos como partes de una unidad social. En otras palabras, podríamos afirmar que los movimientos sociales apelan a la solidaridad entendida como la capacidad de sus miembros para definir y reconocer un sentido del nosotros, y desde ahí compartir y construir una identidad colectiva.

De lo anterior se deriva la relevancia que $B$. De Sousa le atribuye a la solidaridad como fuente y forma de saber, que tendría la posibilidad de enfrentar y contrarrestar las prácticas colonialistas sobre las cuales se ha sustentado históricamente la producción del saber cómo regulación y dominación, las cuales consisten en la ignorancia de la reciprocidad, la incapacidad de concebir al otro como sujeto generador de experiencia social y, por tanto, de conocimiento.

Desde un conocimiento como emancipación, la solidaridad es el saber que los integrantes de un colectivo social generan y movilizan en el proceso mismo de la acción colectiva como construcción social, el cual es inacabado y nos dispone en capacidad de reciprocidad, a través del reconocimiento de la intersubjetividad, para destacar la experiencia del otro como una fuente de aprendizaje mutuo. En esta perspectiva podríamos decir que la solidaridad como forma específica de conocimiento, conlleva una disposición de acogida, lo que significa reconocimiento, encuentro, intercambio de puntos de vista con otros, aspectos vitales para configurar un sentido del nosotros sobre la base de establecer nuevos lazos sociales que representan confianza, lo que hace que emerjan y se sucinten expresiones de resistencia, porque el reconocimiento reciproco del otro se convierte en la razón de cada sujeto para seguir luchando.

Este plano de análisis conlleva la perspectiva de una ecología del reconocimiento, en la medida en que las nuevas formas de solidaridad como conocimiento se proponen descolonizar nuestras mentes para desnaturalizar las diferencias que ocultan jerarquías y que justifican prácticas de exclusión y dominación. De ahíla importancia de resaltar en las diversas experiencias de organización y autogestión comunitarias que se llevan a cabo en nuestros ciudades y campos, las nuevas formas de sociabilidad, los vínculos afectivos y sociales que se tejen en la construcción de las identidades colectivas y en las luchas por el reconocimiento.

\section{La acción colectiva y la construcción de nuevas temporalidades $\mathbf{y}$ territorialidades}

El cambio social que promueven muchos de los movimientos sociales en el continente, se juega, en la actualidad, en la ocupación, defensa y creación de nuevas territorialidades que transciende su carácter geográfico y se constituyen, como lo señala R. Zibechi (2008), en espacios de autoorganización, en espacios de poder en los que se construyen nuevas formas de organización social, como los que gestan movimientos indígenas en Ecuador, Chiapas y Bolivia, los sin tierra del Brasil y los pobladores populares urbanos en nuestras ciudades.

Estudios sobre acciones colectivas de los pobladores populares en ciudades latinoamericanas, como el adelantado por A. Torres (2007), muestran cómo estos movimientos sociales urbanos convierten sus territorios -barrios- en espacios de resistencia y despliegue de su acción política, donde tienen lugar la construcción de vínculos de solidaridad, la conformación de un tejido asociativo, la 
generación de identidades vecinales, y se construyen memorias colectivas urbanas de resistencia, convirtiendo los territorios en espacios de socialización y educación política donde se gestan nuevas utopías, poniendo en evidencia el propósito emancipador de sus emprendimientos, en tanto factor de democratización y expansión de la noción y ejercicio de la ciudadanía.

Podemos concluir este aparte trayendo la siguiente afirmación de A. Torres (2007, p. 19): "los pobladores, más que incorporarse a las ciudades, han sido productores de buena parte de su urbanización, de su economía, de su cultura y de la vida política". De esta manera el análisis de la defensa y creación de nuevas territoriedades como espacios de resistencia y de proyección política por parte de los movimientos sociales, demanda asumir una ecología de las temporalidades y de la tras-escala que nos permitan reconocer otras percepciones del tiempo y del espacio presentes en las experiencias sociales.

\section{Contribuciones y desafíos de la educación popular en la construcción y formación de un conocimiento emancipatorio}

La problematización acerca de la invención social de un conocimiento emancipatorio se ha hecho presente también en la educación popular, y en la actualidad ocupa un lugar central en sus agendas de discusión. Así lo muestra la VII Asamblea General del Consejo de Educación de Adultos de América Latina -CEAAL- realizada en Cochabamba, Bolivia, en agosto de 2008, donde se compartieron distintas miradas acerca de los retos y desafíos que tiene la educación popular en la construcción de paradigmas emancipatorios. Preocupación que se reafirma también con el trabajo investigativo adelantado por A. Torres (2008), donde se expone la trayectoria y los derroteros de la educación popular y su aportes a la construcción de nuevos paradigmas desde la producción del CEAAL entre 2004 y 2008.

Este eje de discusión y proyección que se ha ido consolidando, como lo plantea P. Pontual (2008), se hace necesario en la actualidad para enfrentar y comprender los desafíos impuestos por las trasformaciones en el panorama político mundial y latino- americano, producto de las tensiones derivadas de la hegemonía del modelo neoliberal en la región y las expresiones de resistencia y de democratización que animan los movimientos sociales, los cuales han jugado un papel protagónico en la emergencia de gobiernos democráticos progresistas en algunos países. Podríamos decir que en la comunidad de la CEAAL y de la educación popular, hay acuerdos en destacar que los movimientos sociales son uno de los protagonistas de los principales cambios democráticos que vivimos en América Latina.

Esta constatación suscita una serie de interrogantes para la educación popular, como ¿Cuáles son las nuevas expresiones de movimientos sociales? ¿En torno a qué problemas y/o temas se constituyen los procesos de organización y movilización? ¿Cómo se desarrolla la relación entre los movimientos sociales, las ONGs y los partidos políticos? ¿Cuáles son las tensiones y conflictos existentes entre los movimientos sociales? ¿Es posible la construcción de agendas comunes que promuevan la articulación entre movimientos sociales? Frente a estas y otras preguntas considero que las prácticas de educación popular deben propiciar y profundizar un diálogo abierto y crítico con los MS en torno a estas preocupaciones, que permita ir construyendo conjuntamente procesos de formación, que generen espacios de debate y de intercambio de saberes, y de agendas que potencien la reinvención de un conocimiento como emancipación social. A mi juicio, este diálogo fecundo haría posible, como lo ha señalado B. De Sousa, una retórica dialógica cuyo principio regulador es la práctica argumentativa, donde es posible afianzar la solidaridad como forma de conocimiento, y establecer alianzas y relaciones sociales donde emerjan expresiones de poder y de resistencia.

De ahí se desprende el llamado que nos hace este sociólogo portugués por revitalizar el campo de acción de los educadores populares, el cual debe operar como un movimiento, como una red de acción, promoviendo desde los procesos formativos escenarios donde el conocimiento-emancipación se convierta en un sentido común trasformador de las condiciones de exclusión y dominación, donde su 
potencial ético-político radica en enriquecer, desde nuestras experiencias cotidianas, nuestra relaciones de solidaridad.

Ahora bien, comparto con A. Torres (2008) que esta revitalización de la educación popular no parte de cero, sino que debe sustentarse en el cumulado histórico que como corriente pedagógica crítica y movimiento cultural alternativo ha construido, nutriendo sus postulados en diálogo con otras corrientes y prácticas sociales donde es posible reconocer y potenciar desde las acciones formativas, subjetividades emergentes.

Otro referente valioso al que puede acudir la educación popular para contribuir a la construcción de un conocimiento emancipador y al establecimiento de un intercambio de saberes con los movimientos sociales, es el de la sistematización de prácticas y saberes generados desde las mismas experiencias de formación. Varios de los centros afiliados al CEAAL posen experiencias valiosas que han hecho de la sistematización una modalidad investigativa que genera posibilidades de intercambio de conocimientos, que no se limitan a una reconstrucción descriptiva, sino que posibilitan explorar, reconocer y potenciar los saberes y realidades emergentes con potencial emancipador que puedan contribuir en la generación y fortalecimiento de un pensamiento crítico, como lo muestran procesos adelantados con organizaciones campesinas y acciones de promoción de la equidad de género con organizaciones sociales urbanas.

De lo que se trata es de ampliar y renovar, desde la memoria pedagógica construida por los educadores populares, los referentes interpretativos que han alimentado los discursos y las prácticas educativas, de esta manera, como lo plantea Isabel Vinent (2004),

La educación popular podría estar superando uno de los dilemas teóricos más críticos: su vigencia como planteamiento emancipador ante los paradigmas y contextos actuales. Los contemporáneos cambios paradigmáticos en el campo del conocimiento científico, los cambios contextuales imperantes en el terreno de la política y la economía y los nuevos sujetos emergentes en las luchas populares marcan un punto de no retorno en la historia de la educación popular.

Una de las preocupaciones presentes en los planteamientos de B. De Sousa es cómo propiciar una articulación, un intercambio entre los distintos movimientos sociales que, sin negar las particularidades históricas y contextuales, haga posible el diálogo, por ejemplo, entre el movimiento feminista y el indígena, el campesino y los urbanos, logrando movilizar ciertas agendas comunes concertadas.

Quizás la educación popular pueda constituirse en sí misma, en una práctica pedagógica de traducción, que es entendida por este autor como un proceso intercultural, intersocial, que lo que busca es traducir saberes en otros saberes, traducir prácticas y sujetos de unos a otros en búsqueda de inteligibilidad sin homogeneización. El propósito es saber lo que hay de común entre los movimientos sociales, comprender en qué radican sus distinciones con el ánimo de producir sentidos compartidos que nos permitan comprender, reconocer y visibilizar la diversidad de expresiones que conllevan las luchas sociales, las formas de resistencia, las relaciones de solidaridad que generan.

Para concluir, deseo resaltar que el papel relevante que adquiere en la actualidad la educación popular para crear condiciones de articulación de conocimientos, prácticas y acciones colectivas, y para propiciar alianzas y movilizar consensos entre los sujetos sociales, es significativo en este propósito por reinventar y potenciar la emancipación social.

De ahí que uno de sus desafíos sea propender relaciones y puentes entre las prácticas de educación popular y las acciones colectivas que emprenden los sujetos sociales, con el propósito de acompañar los procesos de organización y movilización social, que como lo plantea B. De Sousa (2007), es algo fundamental, pues cada vez la gente quiere más razones propias para movilizarse, y esto exige una educación popular que debe basarse en el reconocimiento de unas pluralidades despolarizadas que posibiliten una articulación de acciones colectivas cada vez mayor y al mismo tiempo contrarresten ciertos extremismos que han sido nefastos para potenciar 
el conocimiento como emancipación, como son los radicalismos y fundamentalismos identitarios e ideológicos.

Teniendo como referente esta premisa de orientación y fundamentación, la educación popular encarnaría y posibilitaría la propuesta de la ecología de los saberes y de la investigación-acción, permitiendo el diálogo y los intercambios entre el saber científico y los saberes populares, sociales, urbanos, rurales que circulan en la sociedad. Esta pedagogía de la traducción cultural y del intercambio de saberes que trabaja precisamente en la articulación de las

\section{Bibliografía}

De Sousa, B. (2003). Crítica de la razón indolente: contra el desperdicio de la experiencia, Vol. I. España: Editorial Desclee de Brouwer.

(2006). Renovar la teoría crítica y reinventar la emancipación social. Universidad de Buenos Aires, Consejo Latinoamericano de Ciencias Sociales -CLACSO-, Buenos Aires.

(2007). Reinventando la emancipación social. Conferencia, Alianza Francesa, 26 de marzo.

Goldar, M. (2009). La construcción de paradigmas emancipatorios desde los actuales movimientos sociales latinoamericanos. En Revista Latinoamericana de Educación y Política La Piragua (No. 30), 69-79. CEAAL, México.

(I/2009). Los movimientos sociales hoy y los desafíos de la educación popular. En Revista diferencias a partir de la multiplicidad de frentes que se abren y se negocian en cada contexto, se constituye en el eje central para avanzar en la realización y concreción de la propuesta formulada por de B. De Sousa sobre la Universidad Popular de los Movimientos Sociales -UPMS- como una gran red global de conocimientos que propicie un espacio de aprendizaje colectivo y de diálogo recíproco de transformación social entre los movimientos sociales, las ONGs, los científicos sociales, los centros de investigación y los artistas, entre otros interlocutores. DI
Latinoamericana de Educación y Política La Piragua (No. 28), 103-114. CEAAL, México.

Pontual, P. (I/2009). En torno a la agenda de la educación popular en CEAAL. En Revista Latinoamericana de Educación y Política La Piragua (No. 28), 28-31. CEAAL, México.

Torres, A. (2007). Identidad y política de la acción colectiva. Bogotá: Universidad Pedagógica Nacional.

(2009). Educación popular y paradigmas emancipadores. En Revista Latinoamericana de Educación y Política La Piragua (No. 30), 11-31. CEAAL, México.

Zibechi, R. (2008). América Latina: periferias urbanas, territorios en resistencia. Bogotá: Ediciones desde abajo. 